TRANSACTIONS OF THE

AMERICAN MATHEMATICAL SOCIETY

Volume 349, Number 11, November 1997, Pages 4367-4383

S 0002-9947(97)02034-5

\title{
SOME RAMIFICATIONS OF A THEOREM OF BOAS AND POLLARD CONCERNING THE COMPLETION OF A SET OF FUNCTIONS IN $L^{2}$
}

\author{
K. S. KAZARIAN AND ROBERT E. ZINK
}

\begin{abstract}
About fifty years ago, R. P. Boas and Harry Pollard proved that an orthonormal system that is completable by the adjunction of a finite number of functions also can be completed by multiplying the elements of the given system by a fixed, bounded, nonnegative measurable function. In subsequent years, several variations and extensions of this theorem have been given by a number of other investigators, and this program is continued here. A mildly surprising corollary of one of the results is that the trigonometric and Walsh systems can be multiplicatively transformed into quasibases for $L^{1}[0,1]$.
\end{abstract}

1. A curious connection between disparate mathematical ideas occurs in the theory of complete systems of real-valued measurable functions. The first of these notions is due to Boas and Pollard [3], who showed that certain incomplete systems in $L^{2}$ may be transformed into complete systems by multiplying the elements of the system by a (fixed) bounded, nonnegative measurable function.

Subsequently, Talalyan [17], [18] proved that a system $\Phi$ is complete in measure on a set $E$ if and only if, for every positive $\varepsilon, \Phi$ is complete in $L^{2}\left(E_{\varepsilon}\right)$, where $E_{\varepsilon}$ is a measurable subset of $E$ that has measure greater than $|E|-\varepsilon$. In addition, Talalyan showed that if $\Phi$ has these properties, so also does any family obtained from $\Phi$ by deleting a finite number of its members. Later, Goffman and Waterman [6] gave a new proof of this theorem and observed that it is always possible to make certain infinite deletions from a system that is complete in measure so as to leave a residual system that also satisfies this condition.

The somewhat surprising coincidence of these ideas was noted in [15], where the following result was established.

Theorem A. Let $E$ be a measurable set of finite, positive measure, and let $\Phi=$ $\left\{\varphi_{n}: n=1,2, \ldots\right\}$ be a subset of $L^{2}(E)$. Then, the following conditions are equivalent:

(BP) There exists a bounded, measurable function, $m$, such that $\left\{m \varphi_{n}: n=\right.$ $1,2, \ldots\}$ is complete in $L^{2}(E)$;

(M) $\Phi$ is complete in measure on $E$;

Received by the editors March 8, 1995 and, in revised form, July 21, 1995.

1991 Mathematics Subject Classification. Primary 42B65, 42C15, 46B15, 41A30, 41A58.

Key words and phrases. Multiplicative completion, weighted $L^{p}$-spaces, Schauder basis, quasibasis, $M$-basis, approximate continuity.

The first author was supported by DGICYT Spain, under Grant PB94-0149, and also by Grant MVR000 from the I.S.F. 
(T) For every positive $\varepsilon$, there exists $E_{\varepsilon} \subset E$ such that $\left|E_{\varepsilon}\right|>|E|-\varepsilon$, and $\Phi$ is complete in $L^{2}\left(E_{\varepsilon}\right)$.

Although the details are not given in [15], adaptations of the arguments given there can be used to establish the corresponding theorem in which the role of $L^{2}$ is played by any space $L^{p}$, with $1 \leq p<+\infty$. It follows, in particular, that all cofinite, and some coinfinite, subsets of a system that is complete in $L^{p}(E)$ are multiplicatively completable.

In the ensuing years, many investigations of problems related to the foregoing have been undertaken, and the theory continues to be developed along various lines. It is to these matters that the present article is devoted.

2. The following notation and terminology are employed in the sequel. Let $B$ be a Banach space, and let $B^{*}$ be the conjugate space associated therewith. A system $X=\left\{x_{n}: n=1,2, \ldots\right\}$, of elements of $B$, is complete in $B$ iff the closure of the set of all finite linear combinations of elements of $X$ coincides with $B$. If, for every natural number $j, x_{j} \notin c \ell\left(X \backslash\left\{x_{j}\right\}\right)$, then $X$ is termed minimal. This condition is equivalent to the following $[7,264]$ : there exists a conjugate system

$$
X^{*}=\left\{x_{n}^{*}: n=1,2, \ldots\right\} \subset B^{*}
$$

such that

$$
x_{i}^{*}\left(x_{j}\right)=\delta_{i j}, \quad \forall i \in \mathbb{N}, \quad \forall j \in \mathbb{N} .
$$

A set $X^{*} \subset B^{*}$ is total on $B$ if the condition $x^{*}(x)=0, \forall x^{*} \in X^{*}$, implies that $x=0$.

If $X=\left\{x_{n}: n=1,2, \ldots\right\}$ is a minimal, complete subset of $B$, and if its associated conjugate system is total on $B$, then $X$ is a basis in the sense of Markusiewicz, or, simply, an $M$-basis for $B[16,219]$. A system $X=\left\{x_{n}: n=1,2, \ldots\right\}$ is a quasibasis for $B$ iff there exists a system $\left\{y_{n}^{*}: n=1,2, \ldots\right\} \subset B^{*}$ such that, for all $x$ in $B, \sum_{n=1}^{\infty} y_{n}^{*}(x) x_{n}$ converges to $x$ in the norm of $B$. This latter generalization of a Schauder basis, introduced by Gelbaum [5], is a weaker notion, since the associated coefficient functionals need not be uniquely determined [16, 278, 766]. Nevertheless, one can employ the Banach-Steinhaus theorem to show that the operators

$$
S_{n}=\sum_{k=1}^{n} y_{k}^{*}(\cdot) x_{k}, \quad n \in \mathbb{N},
$$

are uniformly bounded in this setting as well.

The Haar functions may be defined in the following manner: For all $t \in[0,1]$, let

$$
h_{1}(t)=1
$$

and for $k=0,1,2, \ldots ; j=1,2, \ldots, 2^{k}$, let

$$
h_{k}^{(j)}(t)= \begin{cases}2^{\frac{k}{2}}, & \text { if } \frac{2 j-2}{2^{k+1}}<t<\frac{2 j-1}{2^{k+1}} ; \\ -2^{\frac{k}{2}}, & \text { if } \frac{2 j-1}{2^{k+1}}<t<\frac{2 j}{2^{k+1}} ; \\ 0, & \text { otherwise; }\end{cases}
$$

and, for $n=2^{k}+j$, let $h_{n}=h_{k}^{(j)}$. 
For each $a \in(0,1)$, let $I_{a}$ be the real function of period 1 defined by the conditions

$$
I_{a}(t)= \begin{cases}\frac{a-1}{a}, & \text { if } t \in[0, a] \\ 1, & \text { if } t \in(a, 1] .\end{cases}
$$

3. In [2], Braun strengthened the theorem of Boas-Pollard and generalized that theorem, so as to include other $L^{p}$-spaces, in the following manner.

Theorem B. Let $E$ be a measurable set of finite positive measure, and let $\Phi=$ $\left\{\varphi_{n}: n=1,2, \ldots\right\}$ be a Schauder basis for some space $L^{p}(E)$, with $1 \leq p<$ $+\infty$. Then, to every natural number $N$ there corresponds a bounded measurable function $m$ such that every element $f$ of $L^{p}(E)$ can be represented by a series $\sum_{k=N+1}^{\infty} a_{k} m \varphi_{k}$ that converges to $f$ in the $L^{p}-$ norm.

In other terminology, Theorem B asserts that $\left\{m \varphi_{k}: k>N\right\}$ is a system of representation for $L^{p}(E)$. Neither does the theorem claim, nor are the arguments originally used to establish this result sufficient to show, that $\left\{m \varphi_{k}: k>N\right\}$ is, again, a Schauder basis for $L^{p}(E)$. Thus, one is led to inquire whether it is always possible to obtain a Schauder basis, from the residual system, by means of this sort of multiplicative transformation.

This question was addressed in the series of monographs [8]-[12], where it was shown that one of three situations may obtain. In the first of these, one may remove any finite number of elements from the basis and the residual system can be multiplicatively transformed into a basis. In [10], it was shown that the Haar system has this property; a characterization of the usable multiplier functions was given; and it was observed that certain coinfinite subsystems of the Haar system could be transformed in this way. At the other end of the spectrum, there are Schauder bases for $L^{p}(E)$ for which no cofinite subsystem can be multiplicatively transformed into a basis for $L^{p}(E)$. The trigonometric and Walsh systems are familiar examples of this type. (See [9], [11], [12].) Finally, there are Schauder bases for which some, but not all, of its cofinite subsets can be multiplicatively transformed into bases.

Taking now a different tack, suppose that the requirement that $\Phi$ be a Schauder basis is replaced by some weaker condition. Then, a variety of interesting propositions of Boas-Pollard type obtain.

Theorem 1. Let $\Phi=\left\{\varphi_{n}: n=1,2, \ldots\right\}$ be a quasibasis for some $L^{p}(E), 1 \leq$ $p<\infty$, with $E$ a measurable set of finite, positive measure. Then, to every natural number, $N$, there corresponds a bounded, measurable function, $m$, such that $\left\{m \varphi_{n}\right.$ : $n>N\}$ is a system of representation for $L^{p}(E)$.

For the proof of this modest assertion, it suffices to repeat, mutatis mutandis, the arguments employed by Braun in his demonstration of Theorem B, but a somewhat more extensive modification of those arguments yields a much stronger result.

Theorem 2. Let $\Phi=\left\{\varphi_{n}: n=1,2, \ldots\right\}$ be a quasibasis for some $L^{p}(E), 1 \leq p<$ $\infty$, with $E$ a measurable set of finite, positive measure. Then, to every nonnegative integer, $N$, there corresponds a bounded, measurable function, $m$, such that $\left\{m \varphi_{n}\right.$ : $n>N\}$ is a quasibasis for each $L^{r}(E), 1 \leq r \leq p$.

Corollary 3. Let $\Phi=\left\{\varphi_{n}: n=1,2, \ldots\right\}$ be a complete, orthonormal system on E, a measurable set of finite, positive measure. Then, there exists a bounded, 
measurable function, $m$, such that $\left\{m \varphi_{n}: n=1,2, \ldots\right\}$ is a quasibasis in each space $L^{r}(E), 1 \leq r \leq 2$.

In particular, the corollary shows that the trigonometric and Walsh systems can be multiplicatively transformed into quasibases for $L^{1}[0,1]$, a result hitherto unknown.

The following proof of Theorem 2 depends upon a proposition of MenshovTalalyan type. (See, for example, [18].)

Lemma 4. Let $\Phi=\left\{\varphi_{n}: n=1,2, \ldots\right\}$ be a quasibasis for some space $L^{p}[0,1]$, with $1 \leq p<\infty$, and let $f \in L^{p}[0,1]$. Then, to each positive real number, $\varepsilon$, and each natural number, $N$, there correspond a measurable set $e$ and a $\Phi$-polynomial, $P=\sum_{k=N}^{M} a_{k} \varphi_{k}$, such that:

$$
\begin{gathered}
e \subset[0,1], \quad \text { and } \quad|e|<\varepsilon ; \\
\|f-P\|_{L^{p}(G)}<\varepsilon, \text { where } G=[0,1] \backslash e ;
\end{gathered}
$$

and

$$
\sup \left\{\left\|\sum_{k=n}^{s} a_{k} \varphi_{k}\right\|_{L^{p}(E)}: N \leq s \leq m\right\}<\varepsilon+\|f\|_{L^{p}(E)},
$$

for every measurable subset, $E$, of $G$.

Proof. The argument employs the following result, due to Fejér (see $[19,49,376]$.)

Lemma C. Let $\beta: \mathbb{R} \rightarrow \mathbb{R}$ be periodic, of period 1 , and let $\left.\beta\right|_{[0,1]} \in L^{p}[0,1]$, for some $p \in[1,+\infty]$. Then, for every $\alpha \in L^{q}[0,1]$, where $p$ and $q$ are conjugates, one has

$$
\lim _{n} \int_{0}^{1} \alpha(t) \beta(n t) d t=\int_{0}^{1} \alpha(t) d t \int_{0}^{1} \beta(t) d t .
$$

Certainly, one may assume, without loss of generality, that $0<\varepsilon<1$.

Let $\Psi=\left\{\psi_{n}^{*}: n=1,2, \ldots\right\}$ be a system of coefficient functionals associated with $\Phi$, and, for each $n$, let $\psi_{n} \in L^{q}[0,1]$ be a representative of $\psi_{n}^{*}$. Then, the $n^{\text {th }}$ partial-sum operator is given by

$$
S_{n}(\cdot)=\sum_{k=1}^{n} \psi_{k}^{*}(\cdot) \varphi_{k}=\sum_{k=1}^{n} a_{k}(\cdot) \varphi_{k},
$$

where

$$
a_{k}(\cdot)=\int_{0}^{1}(\cdot) \psi_{k} d t
$$

Since

$$
\lim _{n}\left\|\alpha-S_{n}(\alpha)\right\|_{p}=0, \quad \forall \alpha \in L^{p}[0,1],
$$

an application of the Banach-Steinhaus theorem yields a positive constant, $C_{p}$, such that

$$
\left\|S_{n}\right\| \leq C_{p}, \quad \forall n \in \mathbb{N}
$$

There exist a natural number $n$ and a corresponding step function

$$
g=\sum_{i=1}^{2^{n}} \gamma_{i} \chi_{\Delta_{n}^{i}}, \text { where } \Delta_{n}^{i}=\left(\frac{i-1}{2^{n}}, \frac{i}{2^{n}}\right), \quad i=1, \ldots, 2^{n},
$$


such that

$$
\|f-g\|_{p}<\frac{\varepsilon}{4}
$$

and

$$
\|g\|_{L^{p}\left(\Delta_{n}^{i}\right)}<\frac{\varepsilon}{8 C_{p}}, \quad i=1, \ldots, 2^{n} .
$$

(For example, one could use an integral mean of $f$ associated with a dyadic partition, of $[0,1]$, of sufficiently high order.)

Let $n_{1}=N$, let

$$
B_{1}=\max \left\{\left\|\varphi_{k}\right\|_{p}: 1 \leq k \leq n_{1}\right\},
$$

and let

$$
\eta_{1}=\frac{\varepsilon}{2^{n+3} n_{1} B_{1}} .
$$

By virtue of Fejér's lemma, there exists a natural number, $s_{1}$, such that $s_{1}>n$, and

$$
\left|b_{k}^{1}\right|=\left|\int_{\Delta_{n}^{1}} g(t) \psi_{k}(t) I_{\frac{\varepsilon}{2}}\left(2^{s_{1}} t\right) d t\right|<\eta_{1}, \quad \forall k \in\left[1, n_{1}\right] .
$$

Let $g_{1}$ be the function defined on $[0,1]$ by the equation

$$
g_{1}(t)=\gamma_{1} \chi_{\Delta_{n}^{1}}(t) I_{\frac{\varepsilon}{2}}\left(2^{s_{1}} t\right),
$$

let $n_{2}$ be a natural number, greater than $n_{1}$, such that

$$
\left\|g_{1}-\sum_{k=1}^{n_{2}-1} a_{k}\left(g_{1}\right) \varphi_{k}\right\|_{p}<\varepsilon 2^{-n-3},
$$

and let

$$
P_{1}=\sum_{k=n_{1}}^{n_{2}-1} a_{k}\left(g_{1}\right) \varphi_{k}
$$

Then,

$$
\begin{aligned}
\left\|g_{1}-P_{1}\right\|_{p} & \leq\left\|g_{1}-\sum_{k=1}^{n_{2}-1} a_{k}\left(g_{1}\right) \varphi_{k}\right\|_{p}+\left\|\sum_{k=1}^{n_{1}-1} a_{k}\left(g_{1}\right) \varphi_{k}\right\|_{p} \\
& <\varepsilon 2^{-n-3}+\left\|\sum_{k=1}^{n_{1}-1} b_{k}^{1} \varphi_{k}\right\|_{p} \\
& \leq \varepsilon 2^{-n-3}+n_{1} \eta_{1} B_{1}<\varepsilon 2^{-n-2} .
\end{aligned}
$$


Writing, for the moment, $a=\frac{\varepsilon}{2}$, one finds that

$$
\begin{aligned}
\int_{\Delta_{n}^{1}}\left|g_{1}\right|^{p} d t & =((1-a) / a)^{p}\left|\gamma_{1}\right|^{p} a\left|\Delta_{n}^{1}\right|+\left|\gamma_{1}\right|^{p}(1-a)\left|\Delta_{n}^{1}\right| \\
& =\left[(1-a)^{p}+a^{p-1}(1-a)\right]\left|\gamma_{1}\right|^{p}\left|\Delta_{n}^{1}\right| / a^{p-1} \leq\left(2 / a^{p-1}\right) \int_{\Delta_{n}^{1}}|g|^{p} d t
\end{aligned}
$$

thus,

$$
\begin{aligned}
\sup \left\{\left\|\sum_{k=n_{1}}^{m} a_{k}\left(g_{1}\right) \varphi_{k}\right\|_{p}^{p}: n_{1} \leq m<n_{2}\right\} \leq 2^{p} C_{p}^{p}\left\|g_{1}\right\|_{p}^{p} \\
\leq\left(2^{p+1} C_{p}^{p} / a^{p-1}\right) \int_{\Delta_{n}^{1}}|g|^{p} d t<\left(2^{2 p} C_{p}^{p} / \varepsilon^{p-1}\right)\left(\varepsilon / 8 C_{p}\right)^{p} \\
\quad=\varepsilon 2^{-p} \leq \varepsilon / 2 .
\end{aligned}
$$

Proceeding inductively, suppose that the polynomials associated with the intervals $\Delta_{n}^{j}, 1 \leq j<i$, have been determined. Let

$$
B_{i}=\max \left\{\left\|\varphi_{k}\right\|_{p}: 1 \leq k \leq n_{i}\right\},
$$

and let

$$
\eta_{i}=\frac{\varepsilon}{2^{n+3} n_{i} B_{i}} .
$$

Another application of Fejér's lemma yields an integer, $s_{i}$, such that

$$
s_{i}>n,
$$

and

$$
\left|b_{k}^{i}\right|=\left|\int_{\Delta_{n}^{i}} g(t) \psi_{k}(t) I_{\frac{\varepsilon}{2}}\left(2^{s_{i}} t\right) d t\right|<\eta_{i}, \quad \forall k \in\left[1, n_{i}\right] .
$$

Following the same course traversed above, one determines a natural number $n_{i+1}$, greater than $n_{i}$, such that

$$
\left\|g_{i}-\sum_{k=1}^{n_{i+1}-1} a_{k}\left(g_{i}\right) \varphi_{k}\right\|<2^{-n-3} \varepsilon
$$

Then, for

$$
P_{i}=\sum_{k=n_{i}}^{n_{i+1}-1} a_{k}\left(g_{i}\right) \varphi_{k}
$$

one has

$$
\left\|g_{i}-P_{i}\right\|_{p}<2^{-n-3} \varepsilon+\eta_{i} n_{i} B_{i}<2^{-n-2} \varepsilon,
$$

and

$$
\sup \left\{\left\|\sum_{k=n_{i}}^{m} a_{k}\left(g_{i}\right) \varphi_{k}\right\|_{p}^{p}: n_{i} \leq k<n_{i+1}\right\} \leq C_{p}^{p}\left\|g_{i}\right\|_{p}^{p}<\frac{\varepsilon}{2} .
$$


Finally, let

$$
P=\sum_{i=1}^{2^{n}} P_{i}
$$

let

$$
e=\left\{t \in[0,1]: \sum_{i=1}^{2^{n}} I_{\frac{\varepsilon}{2}}\left(2^{s_{i}} t\right) \chi_{\Delta_{n}^{i}}(t) \neq 1\right\}
$$

and let

$$
G=[0,1] \backslash e .
$$

From the definition of $I_{\frac{\varepsilon}{2}}$, one has

$$
\left|\left\{t \in \Delta_{n}^{i}: I_{\frac{\varepsilon}{2}}\left(2^{s_{i}} t\right) \neq 1\right\}\right|=\left(\frac{\varepsilon}{2}\right)\left|\Delta_{n}^{i}\right|,
$$

so that

$$
|e|=\sum_{i=1}^{2^{n}}\left(\frac{\varepsilon}{2}\right)\left|\Delta_{n}^{i}\right|<\varepsilon .
$$

Since $g_{i}$ agrees with $g$ on $G$,

$$
\|f-P\|_{L^{p}(G)}=\|(f-g)+(g-P)\|_{L^{p}(G)} \leq \frac{\varepsilon}{2}+\sum_{i=1}^{2^{n}}\left\|g_{i}-P_{i}\right\|<\varepsilon .
$$

As for the third condition, one notes that, for every measurable subset, $E$, of $G$,

$$
\begin{aligned}
& \max \left\{\left\|\sum_{i=1}^{j} P_{i}\right\|_{L^{p}(E)}: 1 \leq j \leq 2^{n}\right\} \leq \max \left\{\left\|\sum_{i=1}^{j}\left(P_{i}-g_{i}\right)\right\|_{L^{p}(E)}: 1 \leq j \leq 2^{n}\right\} \\
& \quad+\left\|\sum_{i=1}^{j} g_{i}-f \chi_{\cup_{i=1}^{j} \Delta_{n}^{i}}\right\|_{L^{p}(E)}+\|f\|_{L^{p}(E)} \\
& <\sum_{i=1}^{j} 2^{-n-2} \varepsilon+\left\|\sum_{i=1}^{j}\left(g_{i}-f\right) \chi_{\Delta_{n}^{i}}\right\|_{L^{p}(E)}+\|f\|_{L^{p}(E)} \\
& \leq \frac{\varepsilon}{4}+\|g-f\|_{p}+\|f\|_{L^{p}(E)}<\frac{\varepsilon}{2}+\|f\|_{L^{p}(E)} ;
\end{aligned}
$$

thus, for $m \geq N$, say $n_{j} \leq m<n_{j+1}$, one has

$$
\left\|\sum_{k=N}^{m} a_{k} \varphi_{k}\right\|_{L^{p}(E)} \leq\left\|\sum_{i=1}^{j} P_{i}\right\|_{L^{p}(E)}+\left\|\sum_{k=n_{j}}^{m} a_{k}\left(g_{j}\right) \varphi_{k}\right\|_{L^{p}(E)}<\varepsilon+\|f\|_{L^{p}(E)} .
$$

Proof of Theorem 2. Without loss of generality, one may assume that $E=[0,1]$.

If $\left\{f_{k}: k=1,2, \ldots\right\}$ is any denumerable subset of $L^{p}[0,1]$, then, by virtue of Lemma 3, one may follow the trail blazed by Braun in order to construct a 
measurable function, $m$, with $0 \leq m(t) \leq 1$, for all $t \in[0,1]$, a double sequence of $\Phi$-polynomials $\left\{P_{k j}\right\}_{k=1, j=k}^{\infty}$,

$$
P_{k j}=\sum_{i=n_{k-1}(j)+1}^{n_{k}(j)} a_{i} \varphi_{i}
$$

with

$$
\begin{aligned}
N & =n_{0}(1)<n_{1}(1)=n_{0}(2)<n_{1}(2)<n_{2}(2)=n_{0}(3) \\
& <\cdots<n_{0}(j)<n_{1}(j)<\cdots<n_{j}(j)=n_{0}(j+1)<\cdots,
\end{aligned}
$$

and a sequence $\left\{D_{n}\right\}_{n=1}^{\infty}$ of measurable subsets of $[0,1]$ such that:

(i) $\left|[0,1] \backslash D_{n}\right|<\delta_{n},\left\{\delta_{n}\right\}_{n=1}^{\infty} \downarrow 0$;

(ii) $\left\|f_{k}-m \sum_{j=k}^{\ell} P_{k j}\right\|_{p} \leq 2^{-\ell}, \forall k, \forall \ell \geq k$;

(iii) $\sup \left\{\left\|m \sum_{i=n_{k-1}(\ell)+1}^{s} a_{i} \varphi_{i}\right\|_{p}: s \leq n_{k}(\ell)\right\} \leq 2^{-\ell+2}$, if $\ell>k$;

and, for every measurable set $F \subset D_{\ell}$,

(iv) $\sup \left\{\left\|m \sum_{i=n_{\ell-1}(\ell)+1}^{s} a_{i} \varphi_{i}\right\|_{L^{p}(F)}: s \leq n_{\ell}(\ell)\right\} \leq 2^{-\ell+1}+\left\|f_{\ell}\right\|_{L^{p}(F)}$;

(v) $\sup \left\{\left\|m \sum_{i=n_{\ell-1}(\ell)+1}^{s} a_{i} \varphi_{i}\right\|_{L^{p}\left([0,1] \backslash D_{\ell}\right)}: s \leq n_{\ell}(\ell)\right\} \leq 2^{-\ell+2}$.

Now let $\left\{f_{k}: k=1,2, \ldots\right\}$ be the Haar system, normalized with respect to the $L^{p}$-norm, and let $\left\{g_{k}: k=1,2, \ldots\right\}$ be the corresponding conjugate system. One associates with $\left\{m \varphi_{k}: k>N\right\}$ the system $\Psi$ whose members are determined in the following manner:

$$
\psi_{i}=a_{i} g_{k}, \text { if } n_{k-1}(\ell)<i \leq n_{k}(\ell), \text { for some } k \text { and } \ell \geq k .
$$

Then, with the functionals defined on $L^{r}[0,1]$ by setting

$$
b_{i}(\cdot)=\int_{0}^{1}(\cdot) \psi_{i} d t, \quad \text { for } i=N+1, N+2, \ldots,
$$

$\left\{m \varphi_{i}: i>N\right\}$ proves to be a quasibasis for $L^{r}[0,1]$, for each $r \in[1, p]$.

The following estimates suffice for the demonstration.

Let $f$ be an arbitrary element of $L^{r}[0,1]$, and let $\sum_{k=1}^{\infty} c_{k}(f) f_{k}$ be the expansion of $f$ in the $p$-normalized Haar system. Since

$$
\left|c_{k}(f)\right|=\left|\int_{0}^{1} f g_{k} d t\right|=\left|\int_{0}^{1}\left[f-\sum_{j=1}^{k-1} c_{j} f_{j}\right] g_{k} d t\right| \leq\left\|f-\sum_{j=1}^{k-1} c_{j} f_{j}\right\|_{r} \cdot\left\|g_{k}\right\|_{r^{\prime}},
$$

and since $\left\{f_{k}: k=1,2, \ldots\right\}$ is a Schauder basis for $L^{r}[0,1]$, it follows that

$$
\lim _{k}\left|c_{k}(f)\right| /\left\|g_{k}\right\|_{r^{\prime}}=0 .
$$

The partial sums $S_{n}(f)$ of the series

$$
\sum_{i=N+1}^{\infty} b_{i}(f) m \varphi_{i}
$$


fall into two disjoint classes according as

$$
n=n_{k}(\ell), \text { or } n_{k-1}(\ell)<n<n_{k}(\ell),
$$

for some natural numbers $k$ and $\ell$ (with $\ell \geq k$ ). One estimates the error made in approximating $f$ by $S_{n}(f)$, for each variety of $n$.

In the first case, if $k<\ell$, then one has

$$
\begin{aligned}
& \left\|f-\sum_{i=N+1}^{n} b_{i}(f) m \varphi_{i}\right\|_{r}=\left\|f-\sum_{j=1}^{k} \sum_{i=j}^{\ell} c_{j} m P_{j i}-\sum_{j=k+1}^{\ell-1} \sum_{i=j}^{\ell-1} c_{j} m P_{j i}\right\|_{r} \\
& \leq\left\|f-\sum_{j=1}^{\ell-1} c_{j} f_{j}\right\|_{r}+\left\|\sum_{j=1}^{\ell-1} c_{j} f_{j}-\sum_{j=1}^{k} \sum_{i=j}^{\ell} c_{j} m P_{j i}-\sum_{j=k+1}^{\ell-1} \sum_{i=j}^{\ell-1} c_{j} m P_{j i}\right\|_{r} \\
& =\left\|f-\sum_{j=1}^{\ell-1} c_{j} f_{j}\right\|_{r}+\left\|\sum_{j=1}^{k} c_{j}\left(f_{j}-\sum_{i=j}^{\ell} m P_{j i}\right)+\sum_{j=k+1}^{\ell-1} c_{j}\left(f_{j}-\sum_{i=j}^{\ell-1} m P_{j i}\right)\right\|_{r} \\
& \leq\left\|f-\sum_{j=1}^{\ell-1} c_{j} f_{j}\right\|_{r}+\sum_{j=1}^{k}\left|c_{j}\right|\left\|f_{j}-\sum_{i=j}^{\ell} m P_{j i}\right\|_{r}+\sum_{j=k+1}^{\ell-1}\left|c_{j}\right|\left\|f_{j}-\sum_{i=j}^{\ell-1} m P_{j i}\right\|_{r} \\
& \leq\left\|f-\sum_{j=1}^{\ell-1} c_{j} f_{j}\right\|_{r}+2^{-\ell+1} \sum_{j=1}^{\ell-1}\left|c_{j}\right|,
\end{aligned}
$$

while, if $k=\ell$, then a similar analysis yields

$$
\left\|f-\sum_{i=N+1}^{n} b_{i}(f) m \varphi_{i}\right\|_{r} \leq\left\|f-\sum_{j=1}^{\ell} c_{j} f_{j}\right\|_{r}+2^{-\ell} \sum_{j=1}^{\ell}\left|c_{j}\right| .
$$

Now,

$$
\left|c_{j}\right|=\left|c_{j}(f)\right|=\left|\int_{0}^{1} f g_{j} d t\right| \leq\|f\|_{r}\left\|g_{j}\right\|_{r^{\prime}}, \quad \frac{1}{r}+\frac{1}{r^{\prime}}=1,
$$

and

$$
\begin{aligned}
\left\|g_{j}\right\|_{r^{\prime}}=\left\|h_{j}\right\|_{p}\left\|h_{j}\right\|_{r^{\prime}} & =\left\|h_{j}\right\|_{\infty}^{2}\left|\Delta_{j}\right|^{\left(\frac{1}{p}+\frac{1}{r^{\prime}}\right)}, \text { where } \Delta_{j} \text { is the support of } h_{j}, \\
& =\left|\Delta_{j}\right|^{\left(\frac{1}{p}+\frac{1}{r^{\prime}}-1\right)}=\left|\Delta_{j}\right|^{1-\left(\frac{1}{q}+\frac{1}{r}\right)} \leq j^{\left(\frac{1}{q}+\frac{1}{r}-1\right)} \leq j ;
\end{aligned}
$$

thus,

$$
2^{-\ell+1} \sum_{j=1}^{\ell}\left|c_{j}\right| \leq 2^{-\ell} \ell(\ell+1)\|f\|_{r}
$$

Finally, if $n_{k-1}(\ell)<n<n_{k}(\ell)$, with $k \in \mathbb{N}, \ell \in \mathbb{N}$ and $k \leq \ell$, then, for $k<\ell$,

$$
\begin{aligned}
\left\|f-S_{n}(f)\right\|_{r} & \leq\left\|f-\sum_{j=1}^{\ell-1} c_{j} f_{j}\right\|_{r}+2^{-\ell} \ell(\ell+1)\|f\|_{r}+\left\|c_{k} \sum_{i=n_{k-1}(\ell)+1}^{n} a_{i} m \varphi_{i}\right\|_{p} \\
& \leq\left\|f-\sum_{j=1}^{\ell-1} c_{j} f_{j}\right\|_{r}+2^{-\ell}[\ell(\ell+1)+4 k]\|f\|_{r} .
\end{aligned}
$$


On the other hand, if $k=\ell$, then

$$
\left\|f-S_{n}(f)\right\|_{r} \leq\left\|f-\sum_{j=1}^{\ell-1} c_{j} f_{j}\right\|_{r}+2^{-\ell} \ell(\ell+1)\|f\|_{r}+\left\|c_{\ell} \sum_{i=n_{\ell-1}(\ell)+1}^{n} a_{i} m \varphi_{i}\right\|_{r} .
$$

Setting

$$
\sigma_{\ell n}=\sum_{i=n_{\ell-1}(\ell)+1}^{n} a_{i} m \varphi_{i},
$$

one has, by virtue of condition (v),

$$
\begin{aligned}
\left\|\sigma_{\ell n}\right\|_{r}^{r} & =\left\|\sigma_{\ell n}\right\|_{L^{r}\left([0,1] \backslash D_{\ell}\right)}^{r}+\left\|\sigma_{\ell n}\right\|_{L^{r}\left(D_{\ell}\right)}^{r} \\
& \leq 2^{(-\ell+2) r}+\left\|\sigma_{\ell n}\right\|_{L^{r}\left(D_{\ell} \cap \Delta_{\ell}\right)}^{r}+\left\|\sigma_{\ell n}\right\|_{L^{r}\left(D_{\ell} \backslash \Delta_{\ell}\right)}^{r} .
\end{aligned}
$$

Now, from condition (iv), one finds that

$$
\begin{aligned}
\left\|\sigma_{\ell n}\right\|_{L^{r}\left(D_{\ell} \backslash \Delta_{\ell}\right)}^{r} & \leq\left\|\sigma_{\ell n}\right\|_{L^{p}\left(D_{\ell} \backslash \Delta_{\ell}\right)}^{r} \\
& \leq\left(2^{-\ell+1}+\left\|f_{\ell}\right\|_{L^{p}\left(D_{\ell} \backslash \Delta_{\ell}\right)}\right)^{r}=2^{(-\ell+1) r},
\end{aligned}
$$

while

$$
\begin{aligned}
\left\|\sigma_{\ell n}\right\|_{L^{r}\left(D_{\ell} \cap \Delta_{\ell}\right)}^{r} & \leq\left\|\sigma_{\ell n}\right\|_{L^{p}\left(D_{\ell} \cap \Delta_{\ell}\right)}^{r} \cdot\left|\Delta_{\ell}\right|^{1-\frac{r}{p}} \\
& \leq 2^{r}\left|\Delta_{\ell}\right|^{1-\frac{r}{p}}\left(2^{(-\ell+1) r}+\left\|f_{\ell}\right\|_{p}^{r}\right)=2^{r}\left|\Delta_{\ell}\right|^{1-\frac{r}{p}}\left(2^{(-\ell+1) r}+1\right)^{p} .
\end{aligned}
$$

Thus,

$$
\begin{aligned}
& \left\|c_{\ell} \sum_{i=n_{\ell-1}(\ell)+1}^{n} a_{i} m \varphi_{i}\right\|_{r}=\left|c_{\ell}\right|\left\|\sigma_{\ell n}\right\|_{r} \\
& \quad \leq\left(2^{(-\ell+2) r+1}+2^{r+p}\left|\Delta_{\ell}\right|^{1-\frac{r}{p}}\right)^{\frac{1}{r}} \cdot o\left(\left|\Delta_{\ell}\right|^{\frac{1}{p}-\frac{1}{r}}\right), \text { as } \ell \rightarrow+\infty
\end{aligned}
$$

and it follows that, for all $r \in[1, p]$,

$$
\lim _{n}\left\|S_{n}(f)-f\right\|_{r}=0 .
$$

4. Continuing along this avenue of investigation, corresponding results are obtainable if a completeness condition is substituted for the basis hypothesis.

Theorem 5. Let $\Phi=\left\{\varphi_{n}: n=1,2, \ldots\right\}$ be a minimal, complete system in some $L^{p}(E), 1 \leq p<\infty$, with $E$ a measurable set of finite, positive measure. Then, to every natural number, $N$, there corresponds a bounded, measurable function, $m$, such that $\left\{m \varphi_{n}: n>N\right\}$ is a minimal, complete system in $L^{p}(E)$.

The demonstration given below depends upon the following result from [11].

Theorem D. Let $E$ be a measurable set of finite, positive measure, let $\left\{f_{n}: n=\right.$ $1,2, \ldots\}$ be a minimal, complete system in some $L^{p}(E), 1 \leq p<+\infty$, and let $\left\{f_{n}^{*}: n=1,2, \ldots\right\}$ be the corresponding conjugate system. If $N$ is a natural number, and if $m$ is a bounded, measurable function, then the system $\left\{m f_{n}: n>N\right\}$ will be complete and/or minimal in $L^{p}(E)$ iff the following conditions (1) and/or (2), are satisfied: 
(1) A function of the form

$$
(1 / m) \sum_{k=1}^{N} a_{k} f_{k}^{*}
$$

will be an element of $L^{q}(E)\left(\frac{1}{p}+\frac{1}{q}=1 ; q=+\infty\right.$, if $\left.p=1\right)$ iff every $a_{k}$ is 0.

(2) To every $n>N$ there corresponds a unique numerical sequence $\left\{a_{k}^{(n)}\right\}_{k=1}^{N}$ such that

$$
g_{n}=(1 / m)\left[\sum_{k=1}^{N} a_{k}^{(n)} f_{k}^{*}+f_{n}^{*}\right]
$$

belongs to $L^{q}(E)$.

Proof of Theorem 5. Let the elements of the system conjugate to $\Phi$ be denoted by $\varphi_{n}^{*}, n \in \mathbb{N}$, and let $F$ be the (measurable) subset of $E$ which contains those points that are simultaneously points of approximate continuity for each $\varphi_{n}^{*}$. Since each $\varphi_{n}^{*}$ is approximately continuous almost everywhere in $E$, one has $|F|=|E|$. Let the natural number $N$ be specified. One defines, inductively, a set $T=\left\{t_{j}: 1 \leq j \leq\right.$ $N\} \subset F$, on which $\left\{\varphi_{1}^{*}, \ldots, \varphi_{N}^{*}\right\}$ is linearly independent, in the following manner:

(i) Let $t_{1}$ be any point of $F$ such that $\varphi_{1}^{*}\left(t_{1}\right) \neq 0$;

(ii) Suppose elements $t_{1}, \ldots, t_{k}$ of $F$ have been chosen so that $\sum_{j=1}^{k} a_{j} \varphi_{j}^{*}\left(t_{i}\right)=0$, $\forall i=1, \ldots, k$, only if each $a_{j}=0$. Let the numbers $b_{j}$ be chosen (uniquely) so that $\theta_{k+1}^{*}=\varphi_{k+1}^{*}+\sum_{j=1}^{k} b_{j} \varphi_{j}^{*}$ satisfies $\theta_{k+1}^{*}\left(t_{j}\right)=0$, for $1 \leq j \leq k$. Since $\int_{F} \varphi_{k+1} \theta_{k+1}^{*} d t=\int_{E} \varphi_{k+1} \varphi_{k+1}^{*} d t=1, \theta_{k+1}^{*}$ is nonzero on a subset of $F$ that has positive measure. Let $t_{k+1}$ be any point of the latter set. It follows, at once, that $\left\{\left(\varphi_{j}^{*}\left(t_{1}\right), \ldots, \varphi_{j}^{*}\left(t_{k+1}\right)\right): 1 \leq j \leq k+1\right\}$ is linearly independent.

For each $n>N$, let

$$
\psi_{n}^{*}=\varphi_{n}^{*}+\sum_{k=1}^{N} a_{k}^{(n)} \varphi_{k}^{*},
$$

where the numbers $a_{k}^{(n)}$ have been determined (uniquely) by the conditions

$$
\psi_{n}^{*}\left(t_{j}\right)=0, \quad 1 \leq j \leq N,
$$

and, for each natural number $n$, let $m_{n}$ be the function given by

$$
m_{n}(t)=\max \left\{\left|\psi_{k}^{*}(t)\right|: N+1 \leq k \leq N+n\right\} .
$$

Then, $m_{n}\left(t_{j}\right)=0, \forall n \in \mathbb{N}, \forall t_{j} \in T$, and every $m_{n}$ is approximately continuous at each $t_{j}$.

Since $\int_{E} \varphi_{N+1}^{*} \varphi_{N+1} d t=\int_{E} \psi_{N+1}^{*} \varphi_{N+1} d t$, and since

$$
1=\int_{E} \varphi_{N+1}^{*} \varphi_{N+1} d t \leq\left\|\varphi_{N+1}^{*}\right\|_{q}\left\|\varphi_{N+1}\right\|_{p},
$$

it follows that $\left|\psi_{N+1}^{*}\right|$, and thus also $m_{1}$, are positive on a subset of $E$ that has positive measure. Let $\lambda_{1}$ be a positive number such that

$$
F_{1}=F \cap\left\{t: m_{1}(t)>\lambda_{1}\right\}
$$

has positive measure, and let

$$
E_{1}=\left(F \backslash F_{1}\right) \cap\left\{t: m_{2}(t) \neq 0\right\} .
$$


If $\left|E_{1}\right|>0$, then one chooses a $\lambda_{2} \in\left(0, \lambda_{1} / 2\right)$ such that

$$
F_{2}=E_{1} \cap\left\{t: m_{2}(t)>\lambda_{2}\right\}
$$

satisfies $\left|F_{2}\right|>\left|E_{1}\right| / 2$. If $\left|E_{1}\right|=0$, one sets $F_{2}=\phi$ and takes $\lambda_{2}=\lambda_{1} / 2$.

Proceeding inductively, suppose that the triples $\left(\lambda_{1}, E_{1}, F_{1}\right), \ldots,\left(\lambda_{n}, E_{n}, F_{n}\right)$ have been defined so that

$$
E_{k}=\left(F \backslash \bigcup_{i=1}^{k} F_{k}\right) \cap\left\{t: m_{k+1}(t) \neq 0\right\}, \quad \text { for all } 1 \leq k \leq n,
$$

and, if $\left|E_{k}\right|=0$, then

$$
F_{k+1}=\phi, \text { and } \lambda_{k+1}=\lambda_{k} / 2,
$$

whereas, if $\left|E_{k}\right|>0$, then $0<\lambda_{k+1}<\lambda_{k} / 2$,

$$
F_{k+1}=E_{k} \cap\left\{t: m_{k+1}(t)>\lambda_{k+1}\right\} \text {, and }\left|F_{k+1}\right|>\left|E_{k}\right| / 2 \text {. }
$$

If $\left|E_{n}\right|=0$, take $\lambda_{n+1}=\lambda_{n} / 2$, and let $F_{n+1}=\phi$. If $\left|E_{n}\right|>0$, choose $\lambda_{n+1} \in$ $\left(0, \lambda_{n} / 2\right)$ such that

$$
F_{n+1}=E_{n} \cap\left\{t: m_{n+1}(t)>\lambda_{n+1}\right\}
$$

satisfies $\left|F_{n+1}\right|>\left|E_{n}\right| / 2$.

Let the function $m_{0}$ be defined, on $E$, by setting

$$
m_{0}(t)=\lambda_{n}, \text { if } t \in F_{n}, \quad \forall n \in \mathbb{N},
$$

and, for those $t$ that lie in $E \backslash \bigcup_{n=1}^{\infty} F_{n}$, let $m_{0}(t)=\lambda_{1}$. A function of the type guaranteed by the theorem will be obtained, in the sequel, by modifying the structure of $m_{0}$ in the neighborhood of the set $T$.

Since each $m_{n}$ is approximately continuous on $T$, and since $m_{n}(t)>\lambda_{n}, \forall t \in$ $\bigcup_{r=1}^{n} F_{r}$, it follows that each $t_{j}$ is a point of density of $F \backslash \bigcup_{r=1}^{n} F_{r}, \forall n \in \mathbb{N}$.

Let $T^{\prime}=\left\{t_{j}: t_{j}\right.$ is a point of density of $\left.F_{0}=\bigcup_{n=1}^{\infty} F_{n}\right\}$, and, if $T^{\prime}$ is nonempty, relabel the elements of $T$ so that $T^{\prime}=\left\{t_{j}: 1 \leq j \leq r\right\}$, and, if $T \backslash T^{\prime}$ is nonempty, let $T \backslash T^{\prime}=\left\{\tau_{j}: 1 \leq j \leq s\right\}$.

Let $2 \delta_{0}=\min \left\{\left|t_{i}-t_{j}\right|: 1 \leq i<j \leq N\right\}$, and let $I=\left(t_{1}-\delta_{0}, t_{1}+\delta_{0}\right)$. Since each $\varphi_{j}^{*}, j=1, \ldots, N$, is approximately continuous at $t_{1}$, there is, for each natural number $n$, a $\delta_{n}^{*}$ in $\left(0, \delta_{0}\right)$ such that

$$
\begin{aligned}
& \left|\left\{t \in F:\left|\varphi_{j}^{*}(t)-\varphi_{j}^{*}\left(t_{1}\right)\right|<\frac{1}{n}\right\} \cap\left(t_{1}-\delta, t_{1}+\delta\right)\right| \\
& \quad>\left(2-\frac{1}{2 N}\right) \delta, \quad \forall \delta \in\left(0, \delta_{n}^{*}\right), \quad j=1, \ldots, N .
\end{aligned}
$$

Let

$$
\begin{aligned}
J_{n}(\delta) & =\bigcap_{j=1}^{N}\left\{t \in F:\left|\varphi_{j}^{*}(t)-\varphi_{j}^{*}\left(t_{1}\right)\right|<\frac{1}{n}\right\} \\
& \cap\left(\left(t_{1}-\delta, t_{1}+\delta\right) \backslash\left(t_{1}-\frac{\delta}{2}, t_{1}+\frac{\delta}{2}\right)\right) .
\end{aligned}
$$

Since $t_{1} \in T^{\prime}$, there is an $\eta_{1}^{*}>0$ such that

$$
\left|F_{0} \cap\left(t_{1}-\eta, t_{1}+\eta\right)\right|>\frac{3}{2} \eta, \quad \forall \eta \in\left(0, \eta_{1}^{*}\right),
$$


Let $\delta_{1}$ be an element of $\left(0, \min \left\{\delta_{1}^{*}, \eta_{1}^{*}\right\}\right)$, let $J_{1}=J_{1}\left(\delta_{1}\right)$, and let

$$
S=\left\{n:\left|F_{n} \cap J_{1}\right|>0\right\} .
$$

Because $F_{0} \cap J_{1}$ has positive measure, it follows that $S$ is nonempty. Let $j_{1}$ be any element of $S$, and let

$$
H_{1}=F_{j_{1}} \cap J_{1} .
$$

Since $F_{0} \backslash \bigcup_{i=1}^{j_{1}} F_{i}$ has density 1 at $t_{1}$, there is an $\eta_{2}^{*}>0$ such that

$$
\left|\left(F_{0} \backslash \bigcup_{i=1}^{j_{1}} F_{i}\right) \cap\left(t_{1}-\eta, t_{1}+\eta\right)\right|>\frac{3}{2} \eta, \quad \forall \eta \in\left(0, \eta_{2}^{*}\right) .
$$

Let $\delta_{2} \in\left(0, \min \left\{\frac{\delta_{1}}{2}, \delta_{2}^{*}, \eta_{2}^{*}\right\}\right)$, and let $J_{2}=J_{2}\left(\delta_{2}\right)$. Then, $\left|\left(F_{0} \backslash \bigcup_{i=1}^{j_{1}} F_{i}\right) \cap J_{2}\right|>0$, from which follows the existence of a natural number, $j_{2}>j_{1}$, such that

$$
H_{2}=F_{j_{2}} \cap J_{2}
$$

has positive measure.

Proceeding inductively, one determines a decreasing sequence $\left\{\varepsilon_{n}\right\}_{n=1}^{\infty}$, of positive numbers, an increasing sequence $\left\{j_{n}\right\}_{n=1}^{\infty}$, of natural numbers, and a sequence $\left\{H_{n}\right\}_{n=1}^{\infty}$, of measurable sets, such that $\lim _{n} \delta_{n}=0 ; H_{i} \cap H_{j}=\phi, \forall i, \forall j, i \neq j$; $\left|H_{n}\right|>0$ and $H_{n} \subset\left(t_{1}-\delta_{n}, t_{1}+\delta_{n}\right), \forall n$; and each of $\varphi_{1}^{*}, \ldots, \varphi_{N}^{*}$ is continuous at $t_{1}$ relative to $G_{1}=\bigcup_{n=1}^{\infty} H_{n}$.

If $p>1$, one defines the function $h_{1}$, on $E$, in the following manner:

$$
h_{1}(t)= \begin{cases}1, & \text { if } t \in E \backslash G_{1} ; \\ \mu_{n}\left|H_{n}\right|^{-\frac{1}{q}}, & \text { if } t \in H_{n}, n=1,2, \ldots ;\end{cases}
$$

where $\mu_{n}=\lambda_{j_{n}}$. Then $h_{1} \in L^{q}(E)$, since

$$
\int_{E} h_{1}^{q} d t=\sum_{n=1}^{\infty} \mu_{n}^{q}+\left|E \backslash G_{1}\right| \leq|E|+\sum_{n=1}^{\infty} \lambda_{n}^{q}<+\infty,
$$

but $h_{1} / m_{0} \notin L^{q}\left(E \cap\left(t_{1}-\varepsilon, t_{1}+\varepsilon\right)\right), \forall \varepsilon>0$, since, if $\delta_{n_{0}}<\varepsilon$, then

$$
\int_{G \cap\left(t_{1}-\varepsilon, t_{1}+\varepsilon\right)}\left(h_{1} / m_{0}\right)^{q} d t \geq \sum_{n=n_{0}}^{\infty} \frac{\left|H_{n}\right|}{\left|H_{n}\right|}=+\infty .
$$

If $p=1$, one takes $h_{1}=1$, so that

$$
h_{1} \in L^{\infty}(E), \text { while } h_{1} / m_{0} \notin L^{\infty}\left(G_{1} \cap\left(t_{1}-\varepsilon, t_{1}+\varepsilon\right)\right), \quad \forall \varepsilon>0 .
$$

Similarly, there exist, for each $j=1, \ldots, r$, a measurable set $G_{j} \subset\left(t_{j}-\delta_{0}, t_{j}+\delta_{0}\right)$, and a measurable function $h_{j}$, such that each of $\varphi_{1}^{*}, \ldots, \varphi_{N}^{*}$ is continuous at $t_{j}$ relative to $G_{j} ; h_{j}(t)=1$, if $t \in E \backslash G_{j} ;\left(m_{0} / h_{j}\right) \chi_{G_{j}}$ is bounded; $h_{j} \in L^{q}(E)$; and $h_{j} / m_{0} \notin L^{q}\left(G_{j} \cap\left(t_{j}-\varepsilon, t_{j}+\varepsilon\right)\right), \forall \varepsilon>0$.

If $T \backslash T^{\prime} \neq \emptyset$, one notes that, for every $j \in\{1, \ldots, s\}$, and for every $\theta>0$, one must have

$$
\limsup _{\theta \rightarrow 0+} \frac{\left|\left(\tau_{j}-\theta, \tau_{j}+\theta\right) \cap\left(F \backslash F_{0}\right)\right|}{2 \theta}=\beta_{j}>0
$$


since, in the contrary case, one would have

$$
\begin{aligned}
1 & =\lim _{\theta \rightarrow 0^{+}} \frac{\left|\left(\tau_{j}-\theta, \tau_{j}+\theta\right) \cap F\right|}{2 \theta} \\
& =\lim _{\theta \rightarrow 0^{+}}\left\{\frac{\left|\left(\tau_{j}-\theta, \tau_{j}+\theta\right) \cap F_{0}\right|}{2 \theta}+\frac{\left|\left(\tau_{j}-\theta, \tau_{j}+\theta\right) \cap\left(F \backslash F_{0}\right)\right|}{2 \theta}\right\} \\
& =\lim _{\theta \rightarrow 0^{+}} \frac{\left|\left(\tau_{j}-\theta, \tau_{j}+\theta\right) \cap F_{0}\right|}{2 \theta}
\end{aligned}
$$

an impossibility, since no $\tau_{j}$ is a point of density of $F_{0}$. Thus, if $\alpha_{j}$ is a fixed element of $\left(0, \beta_{j}\right)$, there will be a decreasing sequence, $\left\{\theta_{n}\right\}_{n=1}^{\infty}$, such that $\theta_{1}<\delta_{0}$, $\lim _{n} \theta_{n}=0$, and

$$
\frac{\left|K_{n}\right|}{2 \theta_{n}}>\alpha_{j}
$$

for all $n$, where

$$
K_{n}=\left(F \backslash F_{0}\right) \cap\left(\left(\tau_{j}-\theta_{n}, \tau_{j}+\theta_{n}\right) \backslash\left(\tau_{j}-\theta_{n+1}, \tau_{j}+\theta_{n+1}\right)\right), \quad \forall n \in \mathbb{N} .
$$

(In general, these sequences will depend upon $j$, of course.)

If $p>1$, one defines the function $h_{r+j}$, on $E$, by setting

$$
h_{r+j}(t)= \begin{cases}\left|K_{n}\right|^{-\frac{1}{q}}, & \text { if } t \in K_{n}, \quad n=1,2, \ldots \\ 1, & \text { otherwise. }\end{cases}
$$

If $p=1$, one lets

$$
h_{r+j}(t)= \begin{cases}\frac{1}{\theta_{n}}, & \text { if } t \in K_{n}, \quad n=1,2, \ldots \\ 1, & \text { otherwise. }\end{cases}
$$

Finally, one sets

$$
m=m_{0} / \prod_{j=1}^{N} h_{j} .
$$

Certainly $m$ is both bounded and measurable. In order to show that $\left\{m \varphi_{n}\right.$ : $n=N+1, \ldots\}$ is a minimal, complete system for $L^{p}(E)$, it suffices to show that conditions (1) and (2) of Theorem D are satisfied.

For the first of these, let $g=\sum_{k=1}^{N} a_{k} \varphi_{k}^{*}$, where $a_{1}, \ldots, a_{N}$ are real numbers, and suppose that $g / m \in L^{q}(E)$. Such a $g$ must vanish on $T^{\prime}$, for otherwise, there would exist a $j$ in $\{1, \ldots, r\}$ and a positive number $\varepsilon$ such that

$$
|g(t)|>\left|g\left(t_{j}\right)\right| / 2>0, \quad \forall t \in G_{j \varepsilon}=G_{j} \cap\left(t_{j}-\varepsilon, t_{j}+\varepsilon\right),
$$

from which would follow

$$
\|g / m\|_{E, q} \geq\|g / m\|_{G_{j \varepsilon}, q} \geq\left(\left|g\left(t_{j}\right)\right| / 2\right)\left\|h_{j} / m_{0}\right\|_{G_{j \varepsilon, q}}=+\infty .
$$

As for the points of $T \backslash T^{\prime}$, if such there be, suppose that, for some $j, 2 \gamma=$ $\left|g\left(\tau_{j}\right)\right|>0$. Let $\left\{\theta_{n}\right\}_{n=1}^{\infty}$ and $\left\{K_{n}\right\}_{n=1}^{\infty}$ be the sequences associated with $\tau_{j}$, in the construction given above. By virtue of the approximate continuity of $g$ at $\tau_{j}$, there is a positive number $\eta$ such that

$$
\left|\{t:|g(t)|>\gamma\} \cap\left(\tau_{j}-\theta, \tau_{j}+\theta\right)\right|>\left(1-\frac{\alpha_{j}}{2}\right)(2 \theta), \quad \forall \theta \in(0, \eta) ;
$$


thus, if $n_{0}$ be chosen so that $\theta_{n_{0}}<\eta$, then

$$
\left|\{t:|g(t)|>\gamma\} \cap K_{n}\right|>\frac{1}{2}\left|K_{n}\right|, \quad \forall n \geq n_{0} .
$$

It follows that, in the case $p>1$,

$$
\int_{E}|g / m|^{q} d t \geq \gamma^{q} \sum_{k=n_{0}}^{\infty}\left|K_{n}\right|^{-1}\left(\frac{1}{2}\left|K_{n}\right|\right)=+\infty
$$

while

$$
\begin{aligned}
\|g / m\|_{E, q} & \geq\|g / m\|_{K_{n}, q}, \quad \forall n \geq n_{0}, \\
& \geq \gamma / \theta_{n} \rightarrow+\infty,
\end{aligned}
$$

if $p=1$. Hence, $g$ vanishes at each point of $T$.

Because the set $\left\{\left(\varphi_{j}^{*}\left(t_{1}\right), \ldots, \varphi_{j}^{*}\left(t_{N}\right)\right): 1 \leq j \leq N\right\}$ is linearly independent, one must have $a_{k}=0, \forall k$, so that condition (1) is satisfied.

A parallel argument shows that if, for some $n>N$ and real numbers $a_{1}, \ldots, a_{N}$,

$$
(1 / m)\left[\varphi_{n}^{*}+\sum_{k=1}^{N} a_{k} \varphi_{k}^{*}\right] \in L^{q}(E)
$$

then

$$
\varphi_{n}^{*}\left(t_{j}\right)+\sum_{k=1}^{N} a_{k} \varphi_{k}^{*}\left(t_{j}\right)=0, \quad \forall j=1, \ldots, N
$$

which conditions ensure the satisfaction of condition (2).

In [13] an analogous result was proved for complete, orthonormal systems in $L^{2}$, and similar questions concerning some specific CONS were investigated in articles [11], [4], and [14]. For the classical orthonormal systems examined in [11], [14], it follows automatically, from the minimality and completeness, in $L^{p}$, of $\left\{m \varphi_{n}: n=N+1, \ldots\right\}$, that the system thus multiplicatively completed is, in fact, an $M$-basis. This is not generally the case, however, as the following proposition shows.

Theorem 6. There exist a system $\left\{\varphi_{n}: n=1,2, \ldots\right\}$ that is an $M$-basis for every space $L^{p}[0,1], 1 \leq p<\infty$, and a bounded, measurable function, $m$, such that $\Psi=\left\{m \varphi_{n}: n=2, \ldots\right\}$ is minimal, complete in $L^{p}[0,1]$, for all $p$ in $[1, \infty)$, while $\Psi$ is an $M$-basis for none of these spaces.

Proof. The demonstration makes use of a construction suggested by Gelbaum [5]. (See also $[16,236]$.) Let $\left\{\alpha_{j}\right\}_{j=0}^{\infty}$ be an increasing sequence of real numbers, such that $\alpha_{0}=1$, and

$$
\beta_{j}=1 / \alpha_{j-1}-1 / \alpha_{j}=1 /(j+1)^{3}, \quad \forall j \in \mathbb{N},
$$

and let

$$
\varphi_{n}=\sum_{j=1}^{n} 2 \alpha_{j-1} \cos \pi(j-1)(\cdot), \quad \forall n \in \mathbb{N} .
$$


Since $\Theta=\{\cos \pi j(\cdot): j=0,1, \ldots\}$ is complete in $L^{p}[0,1]$, for every $p$ in $[1, \infty)$, it follows that $\Phi=\left\{\varphi_{n}: n=1,2, \ldots\right\}$ is complete in each of those spaces. If $\Phi^{*}=\left\{\varphi_{n}^{*}: n=1,2, \ldots\right\}$, where

$$
\varphi_{n}^{*}=\left(1 / \alpha_{n-1}\right) \cos \pi(n-1)(\cdot)-\left(1 / \alpha_{n}\right) \cos \pi n(\cdot), \quad \forall n \in \mathbb{N},
$$

then $\left\{\Phi, \Phi^{*}\right\}$ is a biorthogonal system; thus, $\Phi$ is minimal in each space $L^{p}[0,1]$, $1 \leq p<\infty$. Then,

Now suppose that $f$ is an integrable function such that $\varphi_{n}^{*}(f)=0, \forall n \in \mathbb{N}$.

$$
\left(1 / \alpha_{n-1}\right) \int_{0}^{1} f(t) \cos \pi(n-1) t d t-\left(1 / \alpha_{n}\right) \int_{0}^{1} f(t) \cos \pi n t d t=0, \quad n=0,1, \ldots,
$$

so that, for each nonnegative integer $k$,

$$
\left(1 / \alpha_{k}\right) \int_{0}^{1} f(t) \cos k \pi t d t=\left(1 / \alpha_{n}\right) \int_{0}^{1} f(t) \cos \pi n t d t, \quad \forall n>k .
$$

By virtue of the Riemann-Lebesgue lemma, it follows that

$$
\int_{0}^{1} f(t) \cos k \pi t d t=0, \quad \forall k=0,1, \ldots
$$

thus, from the totality of $\Theta$ on $L[0,1]$, it follows that $f(t)=0$, a.e. Hence, $\Phi^{*}$ is total in $L^{1}[0,1]$ and, thus, also in each $L^{p}[0,1]$ with $1<p<\infty$.

Let $m$ be the identity function on $[0,1]$, and let $\Psi=\left\{m \varphi_{n}: n=2, \ldots\right\}$. Since $a \varphi_{1}^{*} / m \in L^{p}[0,1]$ iff $a=0$, and, since, for each $n>1,(1 / m)\left[a^{(n)} \varphi_{1}^{*}+\varphi_{n}^{*}\right] \in L[0,1]$ iff

$$
a^{(n)}\left(1-1 / \alpha_{1}\right)+1 / \alpha_{n-1}-1 / \alpha_{n}=0,
$$

iff

$$
a^{(n)}=-\beta_{n}\left(1-1 / \alpha_{1}\right)^{-1}=-8 \beta_{n},
$$

an application of Theorem $\mathrm{D}$ shows that $\Psi$ is minimal complete in $L^{p}[0,1]$, for every $p$ in $[1, \infty)$. Moreover, for $i \geq 2, j \geq 2$,

$$
\int_{0}^{1}\left[t \varphi_{i}(t)\right]\left[(1 / t)\left(\varphi_{j}^{*}(t)-8 \beta_{j} \varphi_{1}^{*}(t)\right] d t=\delta_{i j}\right.
$$

thus, the system conjugate to $\Psi$ is $\Psi^{*}=\left\{\psi_{n}^{*}: n=2, \ldots\right\}=\left\{(1 / m)\left(\varphi_{n}^{*}-8 \beta_{n} \varphi_{1}^{*}\right)\right.$ : $n=2, \ldots\}$.

Since $\alpha_{n}<2$, for every $n$, it follows that $\sum_{n=2}^{\infty} \beta_{n} \varphi_{n}$ converges in each space $L^{p}[0,1], 1 \leq p<\infty$. Denoting the sum of this series by $f$, one finds that

$$
\int_{0}^{1}\left[8 f(t)-\varphi_{1}(t)\right] t \psi_{n}^{*}(t) d t=0, \quad \forall n \geq 2 ;
$$

thus, if $\Psi^{*}$ were total in $L^{p}[0,1]$, it would follow that

$$
8 f(t)-\varphi_{1}(t)=0 \text {, a.e. }
$$

Since this is impossible, by virtue of the minimality of the system $\Phi$, in each space $L^{p}[0,1], 1 \leq p<\infty$, it cannot be the case that $\Psi$ is an $M$-basis for any of the $L^{p}$-spaces. 


\section{REFERENCES}

1. Stefan Banach, Théorie des Opérations Linéaires, Monografje Matematyczne, Warszawa, 1932; latest reprint, Éditions Jacques Gabay, Sceaux, 1993. MR 97d:01035

2. Ben-Ami Braun, On the multiplicative completion of certain basic sequences in $L^{p}, 1<p<$ $\infty$, Trans. Amer. Math. Soc. 176 (1973), 499-508. MR 47:2331

3. R. P. Boas and Harry Pollard, The multiplicative completion of sets of functions, Bull. Amer. Math. Soc. 54 (1948), 518-522. MR 10:189b

4. V. F. Gaposhkin, Trigonometric Cesàro bases in the spaces of functions integrable with power weight, Analysis Math. 8 (1982), 103-124. MR 82c:42017

5. Bernard R. Gelbaum, Notes on Banach spaces and bases, An. Acad. Brasil. Ci. 30 (1958), 29-36. MR 20:5419

6. Casper Goffman and Daniel Waterman, Basis sequences in the space of measurable functions, Proc. Amer. Math. Soc. 11 (1960), 211-213. MR 22:2886

7. Stefan Kaczmarz and Hugo Steinhaus, Theorie der Orthogonalreihen, Monografje Matematyczne, Warszawa-Lwów, 1935; reprint, Chelsea, New York, 1951.

8. K. S. Kazarian, On the multiplicative completion of basic sequences to bases in $L^{p}, 1 \leq p<\infty$, Doklady Akad. Nauk Arm. SSR 62 (1976), 203-209 (Russian). MR 55:3675

9. - On the multiplicative completion of some incomplete orthonormal systems to bases in $L^{p}, 1 \leq p<\infty$, Analysis Math. 4 (1978), 37-52 (Russian). MR 58:2001

10. (1982), 227-249. MR 84d:42037

11. 13 (1978), 315-351 (Russian). MR 80j:42038

12. - On multiplicative completion of uniformly bounded orthonormal systems to basis in $L^{p}, p \geq 1$, Izv. Akad. Arm. SSR Ser. Math. 18 (1983), 344-361; English trans. in Soviet Jour. Contemp. Math. Anal. 18 (1983). MR 86a:42031

13. - Improving a theorem of $R$. Boas and H. Pollard on the multiplicative completion, Izv. Akad. Arm. SSR Ser. Math. 25 (1990), 409-412; English trans. in Soviet Jour. Contemp. Math. Anal. 25 (1990). MR 92g:42019

14. _ Summability of generalized Fourier series and Dirichlet's problem in $L^{p}(d \mu)$ and weighted $H^{p}$-spaces $(p>1)$, Analysis Math. 13 (1987), 173-197. MR 89b:42023

15. J. J. Price and Robert E. Zink, On sets of functions that can be multiplicatively completed, Ann. Math. 82 (1965), 139-145. MR 31:1349

16. Ivan Singer, Bases in Banach Spaces, II, Springer-Verlag, Berlin, Heidelberg, New York, 1981. MR 82k: 46024

17. A. A. Talalyan, On the convergence almost everywhere of subsequences of partial sums of general orthogonal series, Izv. Akad. Nauk Arm. SSR Izv. Fiz-Mat. Estest. Tehn Nauki 10 (1957), 17-34. MR 19:742b

18. - The representation of measurable functions by series, Uspekhi Math. Nauk 15 (1960), no. 5, 77-142 (Russian); English translation in Russian Math. Surveys 15 (1960), no. 5, 77136. MR 23:A2704

19. A. Zygmund, Trigonometric Series, 2nd ed., Vol. I, Cambridge Univ. Press, London, 1959. MR 21:6498

Departamento de Matemáticas, C-XV, Universidad Autónoma de Madrid, 28049 MADRID, SPAIN

Institute of Mathematics of the National Academy of Sciences, av. Marshal Bagramian, 24-B, 375019 Erevan, Republica Armenia

E-mail address: kazaros.kazarian@uam.es

Department of Mathematics, Purdue University, 1395 Mathematical Sciences BuildING, West Lafayette, Indiana 47907-1395, USA

E-mail address: zink@math.purdue.edu 